\title{
Small leucine-rich proteoglycans in the vertebrae of Atlantic salmon Salmo salar
}

\author{
Mona E. Pedersen ${ }^{1, *}$, Elisabeth Ytteborg ${ }^{1}$, Achim Kohler ${ }^{1,2}$, Grete Baeverfjord ${ }^{1}$, \\ Grethe Enersen ${ }^{1}$, Bente Ruyter ${ }^{1}$, Harald Takle ${ }^{1,3}{ }^{3}$ Kirsten O. Hannesson ${ }^{1}$ \\ ${ }^{1}$ Nofima AS, Pb 6122, 9291 Tromsø, Norway \\ ${ }^{2}$ Centre for Integrative Genetics (CIGENE), Department of Mathematical Sciences and Technology, \\ Norwegian University of Life Sciences, 1432 Ås, Norway \\ ${ }^{3}$ AVS Chile SA, Casilles 300, Puerto Varas, Chile
}

\begin{abstract}
We analysed the distribution and expression of the small leucine-rich proteoglycans (SLRPs) decorin, biglycan and lumican in vertebral columns of Atlantic salmon Salmo salar L. with and without radiographically detectable deformities. Vertebral deformities are a reoccurring problem in salmon and other intensively farmed species, and an understanding of the components involved in the pathologic development of the vertebrae is important in order to find adequate solutions to this problem. Using immunohistology and light microscopy, we found that in nondeformed vertebrae biglycan, lumican and decorin were all expressed in osteoblasts at the vertebral growth zones and at the ossification front of the chondrocytic arches. Hence, the SLRPs are expressed in regions where intramembranous and endochondral ossification take place. In addition, mRNA expression of biglycan, decorin and lumican was demonstrated in a primary osteoblast culture established from Atlantic salmon, supporting the in vivo findings. Transcription of the SLRPs increased during differentiation of the osteoblasts in vitro and where lumican mRNA expression increased later in the differentiation compared with decorin and biglycan. Intriguingly, in vertebral fusions, biglycan, decorin and lumican protein expression was extended to transdifferentiating cells at the border between arch centra and osteoblast growth zones. In addition, mRNA expression of biglycan, decorin and lumican differed between non-deformed and fused vertebrae, as shown by quantitative PCR (qPCR). Western blotting revealed an additional band of biglycan in fused vertebrae which had a higher molecular weight than in non-deformed vertebrae. Fourier-transform infrared (FTIR) spectroscopy revealed more spectral focality in the endplates of vertebral fusions and significantly more non-reducible collagen crosslinks compared with non-deformed vertebrae, thus identifying differences in bone structure.
\end{abstract}

KEY WORDS: Atlantic salmon · Bone development $\cdot$ Skeletal deformities $\cdot$ SLRP $\cdot$ Vertebrae

\section{INTRODUCTION}

The vertebral column is the defining character in all vertebrates. Thus, spinal disorders are a major cause of disability known to cause severe pain and discomfort in humans and also ethical and economic problems for intensively farmed animals. In Atlantic salmon Salmo salar, 2 well-characterized mechanisms are responsible for bone formation: the compact bone of the amphicoel and trabeculae is formed directly by intramembranous ossification, and the cartilaginous template is replaced by bone in the arch centra through endochondral ossification. Spinal fusions are one of many skeletal deformities observed in Atlantic salmon (Witten et al. 2009) and may be induced by increased water temperature during the early developmental stages in intensive aquaculture production (Baeverfjord 2003). The pathology and molecular 
mechanism involved in developing vertebral fusions has been partly characterized (Witten et al. 2005, 2006, Ytteborg et al. 2009, 2010b, Pedersen et al. 2011). In summary, these studies showed that ectopic bone formation was a key event in the final stage of developing vertebral fusions, suggesting that a metaplastic shift is involved in the process (Witten et al. 2005, Ytteborg et al. 2010a).

In mammals it is well established that cartilage and bone cellular activity largely depends on the interaction with the extracellular matrix (ECM) (for reviews, see Waddington et al. 2003, Gentili \& Cancedda 2009, Pihlajaniemi et al. 2009). Bone ECM consists of several different components, such as collagens, glycoproteins and proteoglycans (PGs), which build up the template where mineralization takes place. In addition, these components play a pivotal role in proliferation and differentiation by interacting with growth factors and enzymes. Collagen spacing, arrangement and crosslinking are thus important for the availability of mineralization and formation of crystals (Gerstenfeld et al. 1993, Yamauchi \& Katz 1993, Pornprasertsuk et al. 2004, 2005). We have previously demonstrated reduced collagen transcription, together with reduced transcription of mineralization markers such as osteocalcin and alkaline phosphatase, in vertebral fusions (Ytteborg et al. 2010a), indicating that the ECM of vertebral fusions is altered. Moreover, cellular events such as proliferation of the osteoblasts and maturation of the chondrocytes is disturbed (Ytteborg et al. 2010a), suggesting that altered ECM also affects cellular events in Atlantic salmon.

Significant attention has been drawn to the ECM components named small leucine-rich proteoglycans (SLRPs) in mammals, due to their involvement in tissue development, homeostasis and regeneration (Iozzo 1998, 1999, Schaefer \& Iozzo 2008). Members of this family are classified into 5 distinct subfamilies (Nikitovic et al. 2012), and they contain several leucinerich loops that fold into a helical structure, where the leucine-rich loops are thought to bend outward, facilitating collagen binding to the protein core, thus contributing to the regulation of collagen fibrillogenesis (Kalamajski \& Oldberg 2010). The SLRPs carry glycosaminoglycan side chains of varying numbers and types, and their specificity is restricted to different collagen types and to different positions on the same collagen fibers. Hence, they play specific roles in fibrillogenesis, forming different collagen network and fibril bundle phenotypes (Svensson et al. 1995, Danielson et al. 1997, Matheson et al. 2005, Kalamajski \& Oldberg 2010). Studies have also demonstrated a direct role of decorin in regulating collagen crosslinking in vitro (e.g. Seidler et al. 2005). The 3 members of the SLRP family, decorin, biglycan and lumican, have all been detected in long bones, with specific distributions in bone and cartilage (Bianco et al. 1990, Takagi et al. 2000, Alini \& Roughley 2001, Raouf et al. 2002, Yang et al. 2012). They seem to play different roles, where a targeted disruption of the decorin gene alone does not reveal a osteopenia phenotype as in the case of the biglycan gene (Danielson et al. 1997), but when genes for decorin and biglycan are disrupted, a much more severe phenotype than for biglycan alone is observed (Corsi et al. 2002). Compensatory mechanism among the different SLRP family members in the knee joint have also been demonstrated (Nuka et al. 2010). Biglycan plays a role in osteoblast mineralization through bone morphogenetic protein (BMP) regulation in vitro (Parisuthiman et al. 2005), and its expression indicated a more dominant role in the mineralization process compared with decorin, both in vivo (Bianco et al. 1990) and in vitro (Boskey et al. 1997). In addition, the roles of decorin and lumican in the maturation and mineralization of osteoblasts have been described in vitro (Raouf et al. 2002, Mochida et al. 2003). In biglycan knock-out pre-osteoblasts, biglycan was found to regulate the activity of osteoblastic progenitors through sets of genes associated with the cell cycle and differentiation (Chen et al. 2005). The increasing list of different functions of SLRPs and their expression in mammalian bone indicates that SLRPs may play an important role in bone development and deformities in fish as well.

At present, no studies exist on SLRPs in the vertebral column of Atlantic salmon, except the demonstration of decorin at the mRNA level (Ytteborg et al. 2009). In this experiment we examined the expression of biglycan, decorin and lumican in the vertebral column of Atlantic salmon in order to reveal their possible functions in the bone development of teleosts. We further analyzed their expression in spinal fusions and included Fourier-transform infrared (FTIR) analysis in order to characterize the bone structure (mineral/matrix, carbonation, crystallinity and collagen crosslinking).

\section{MATERIALS AND METHODS}

\section{Experimental groups and sampling}

The fish breeding was carried out at Nofima Marin research station (Sunndalsøra, Norway) in 2007 with 
Atlantic salmon eggs originating from SalmoBreeds. A long-term elevation of temperature during early juvenile development was used in order to induce vertebral fusions. The experiment design and setup is described in Ytteborg et al. (2010a). Briefly, based on radiographic examinations, vertebrae were divided into different groups. The non-deformed (ND) group had no observable morphological changes in the vertebral centra or in the intervertebral space. Samples characterized as fused (FS) ranged from incomplete fusions to complete fusions (Fig. 1). Five vertebral bodies $(\sim 1 \mathrm{~cm})$ were carefully dissected from 12 individuals per categorized group, snap-frozen in liquid nitrogen and stored at $-80^{\circ} \mathrm{C}$ before further analysis. Samples for immunohistochemistry were fixed in $4 \%$ paraformaldehyde for $24 \mathrm{~h}$ at $4^{\circ} \mathrm{C}$ and further subjected to metyl methacrylate embedding according to the procedure described by Torgersen et al. (2009).

\section{RNA isolation, cDNA synthesis and real-time PCR}

Frozen vertebrae from 24 individuals ( $\mathrm{n}=12$ from each group) were homogenized in liquid nitrogen before total RNA was isolated using Trizol (Invitrogen) and reagents from the RNAeasy Micro to Midi Kit (Qiagen). All samples were DNase treated (DNase I, Invitrogen), and the RNA quality was assessed by $1 \%$ agarose gel. One microgram of RNA was subjected to reverse transcriptase (RT)-reaction using the Taqman Gold RT-PCR kit (Applied Biosystems). The samples were diluted $5 \times$ before application of samples (in triplicates) to real-time PCR analysis in an ABI Prism 7900HT Sequence Detection System (Applied Biosystems), using the TaqMan 100 rx PCR Core Master Kit (Applied Biosystems). At first, uracil-N glycosylase (UNG) treatment at $50^{\circ} \mathrm{C}$ for $2 \mathrm{~min}$ and UNG inactivation at $95^{\circ} \mathrm{C}$ for $10 \mathrm{~min}$ was performed, followed by amplification of cDNA by 40 two-step cycles $\left(15 \mathrm{~s}\right.$ at $95^{\circ} \mathrm{C}$ for denaturation of DNA, 1 min at $60^{\circ} \mathrm{C}$ for primer annealing and extension). Cycle threshold $\left(C_{\mathrm{t}}\right)$ values were obtained graphically (Applied Biosystems, Sequence Detection System, software version 2.2). Gene expression was normalized to elongation factor 1 alpha (EF-1 $\alpha$ ) and $\Delta C_{\mathrm{t}}$ values were calculated. Comparison of gene expression between samples (ND and FS) was derived from subtraction of $\Delta C_{\mathrm{t}}$ values between the 2 samples to give a $\Delta \Delta C_{\mathrm{t}}$ value, and relative gene expression was calculated as $2^{-\Delta \Delta C_{\mathrm{t}}}$ normalized to ND. The primers and TaqMan probes (5'-labeled-6-FAM and 3' Quencher TAMRA) (Table 1) for real-time PCR amplification of decorin (GenBank accession no. NM001173562.1), biglycan (GenBank accession no. FJ79999.1), lumican (Gen Bank accession no. NM001140062.1) and internal standard EF-1 $\alpha$ (GenBank accession no. AF321836) were designed using the Primer Express Program (Applied Biosystems).
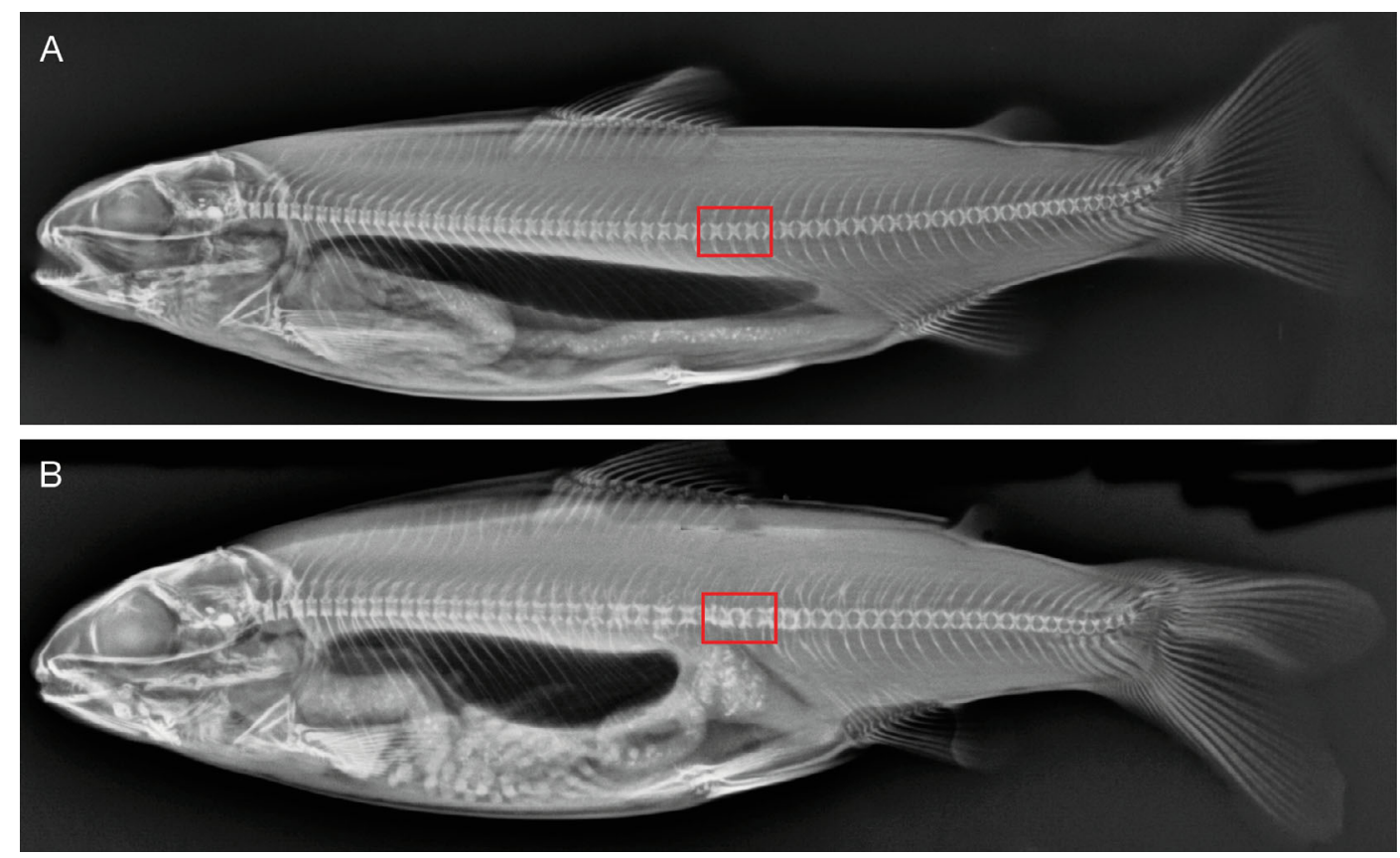

Fig. 1. X-ray of fishes categorized as (A) non-deformed and (B) fused. Sample areas taken for further analysis are marked with a red rectangle 
Table 1. Primer and probe sequences

\begin{tabular}{|ll|}
\hline \multirow{2}{*}{ Gene } & Nucleotide sequence \\
\hline \multirow{2}{*}{ Becorin } & Fwd-5'-CAT TCC CCT GGG CAA GCT-3' \\
& Rev-5' - TTG GCA GGC ATT TCC TTG AG-3' \\
& P-5'-CAG CGC CTC TAC CTC TCC AAG AAC C-3' \\
& Fwd-5'-CAG GTG GTC TAC CTC CAT TCC A-3' \\
& Rev-5'-AAA GCC TCT TGG GCA GAA GTC-3' \\
& P-5'-CAG CAT CAG CAA GGT GGG AGT GGA-3' \\
Lumican & Fwd-5'-AAG TTC TCT GGC CCT CTG AAC TAC-3' \\
& Rev-5'-AGG TCG GCA TGT GTG AGG TT-3' \\
& P-5'-ACT GAA GCA CCT GCG TCT GG-3' \\
EF-1 $\alpha$ & Fwd-5'-GCC AGA TCT CCC AGG GCT AT-3' \\
& Rev-5'-TGA ACT TGC AGG CGA TGT GA-3' \\
& P-5'-CCC CTG TGC TGG ATT GCC ATA C-3' \\
\hline
\end{tabular}

\section{Protein extraction and western blots}

Proteins were isolated from the residual fractions after RNA isolation by Trizol, according to the manufacturer's protocol, and protein concentrations were measured with the RC DC Protein Assay (Bio-Rad). Thereafter, $20 \mu \mathrm{g}$ of protein from each individual of both groups ( $\mathrm{n}=5$ from each group) were solubilised in $6 \mathrm{M}$ urea added to NuPAGE LDS sample buffer (Invitrogen) containing NuPAGE sample reducing agent (Invitrogen), and heated for $10 \mathrm{~min}$ at $70-80^{\circ} \mathrm{C}$ before separation by SDS-gel electrophoresis. The SDS-gel electrophoresis was carried out using 4-12\% BisTris gels (Invitrogen), SDS-PAGE NuPAGE MOPS SDS running buffer (Invitrogen) and the Novex Xcell II apparatus (Invitrogen). Transfer of the proteins to nitrocellulose membrane was them carried out by a dry blotting system using iBlot nitrocellulose gel transfer stacks (Invitrogen) and an iBlot gel transfer device (Invitrogen). Non-specific binding sites were blocked using $5 \%$ teleostan gelatine (Sigma) in 0.1 M Tris-saline, pH 7.4. The nitrocellulose membrane was then probed with the polyclonal antibodies decorin (ab35378), lumican (ab70191) and biglycan (ab20033) (Abcam) diluted 1:5000 in Tris-buffered saline (TBS) added to $0.5 \%$ teleostan gelatine. These antibodies are specific to teleosts (Tingbø et al. 2012). Alkaline phosphatise (AP) conjugated secondary antibodies (anti-mouse IgG(H+L), Promega) diluted 1:7500 were then applied to the membranes. Immunoreactive bands were revealed with Novex AP (BCIP/NBT) color development substrate (Invitrogen), and the reaction was stopped by distilled water.

\section{Immunohistological staining}

Serial sections of $6 \mu \mathrm{m}$ thickness were prepared in parasagittal orientation from vertebral columns of 8 individuals $(n=4$ from each group), starting at the periphery and ending at the middle plane of the vertebrae using a Microm HM 355S (Thermo Fisher Scientific), and mounted on poly-L-lysine-coated glass slides. Resin removal was carried out by successive incubations in xylene (1 h), 2-methoxyethylacetate $(1 \mathrm{~h})$ and acetone $(10 \mathrm{~min})$. The slides were then rehydrated in ethanol series and rinsed for $5 \mathrm{~min}$ in diethylpyrocarbonate-treated distilled water $\left(\mathrm{dH}_{2} \mathrm{O}\right)$ before the analyses. Immunostaining was performed using a DakoCytomation EnVision+ System-HRP(DAB) kit (Dako) according the manufacturer's recommendation and sheep polyclonal decorin antibody (1:1000), sheep polyclonal biglycan antibody (1:100) and sheep polyclonal lumican antibody (1:100), in phosphate-buffered saline (PBS) with added 5\% teleost gelatin (Sigma). Unspecific binding sites were blocked using $5 \%$ teleost gelatin (Sigma) in PBS. Non-specific binding of the primary antibodies was controlled by substitution of the primary antibody with rabbit whole serum (X0933, Dako) diluted 1:1000 in PBS. Non-specific binding of the secondary antibodies was tested by replacing the latter with dilution buffer (PBS). Sections were counterstained with hemotoxylin (Riedel de Haen) combined with erythrosine B (Aldrich-Chemie) to outline tissue architecture. In brief, sections were stained in hematoxylin for 5-10 min, washed in water for $20 \mathrm{~min}$, and fixed in $0.25 \%$ hexamine for $3 \mathrm{~min}$ before being stained in erythrosine B for $4 \mathrm{~min}$. Finally, sections were dehydrated in ascending concentrations of alcohol, cleared in xylol and mounted in Eukitt. A spot RT Color Camera (Diagnostic Instruments) was used to photograph the sections in a Leica DMLB microscope (Leica Microsystems Nussloch).

\section{FTIR analysis}

For FTIR microscopy, cross-sections $(8 \mu \mathrm{m})$ of the vertebral columns collected were cut in a cryostat (Leitz 1720 Digital, Leica Instruments) and mounted on ZnSe slides and stored overnight in a desiccator at room temperature before measurements were taken. The FTIR measurements were carried out with an IRscope II combined with an Equinox 55 FT-IR spectrometer (both Bruker Optics) using OPUS-NT soft- 
ware version 4.0. Spectra were collected from the vertebral end plate in transmission mode in the frequency range 4000 to $750 \mathrm{~cm}^{-1}$ using a mercury cadium telluride (MCT) detector. A background spectrum of the ZnSe slide was recorded before each spectrum was measured in order to correct for water and $\mathrm{CO}_{2}$. Four spectra from each vertebral endplate section and together 4 sections from non-deformed and deformed vertebral columns were collected, resulting in 16 spectra per fish. Spectra were collected from 3 fish belonging to the 2 groups to be compared. The spectra were further preprocessed using extended multiplicative signal corrections (EMSC) in The Unscrambler (Camo Process AS, version 9.2) to remove multiplicative baseline effects. The mineral/matrix ratio, carbonation, crystallinity and collagen crosslinks were calculated according to Boskey \& Camacho (2007) using MATLAB V.7.10 (The Mathworks) and in-house developed algorithms in MATLAB V.7.10.

\section{Osteoblast cell culture isolated from Atlantic salmon muscle precursors}

Myosatellite cells were isolated from Atlantic salmon as described by Koumans et al. (1990), using the modification for Atlantic salmon as described by Vegusdal et al. (2004). Cells were allowed to adhere overnight in an L-15 medium containing $10 \%$ fetal bovine serum (FBS), $2 \mathrm{mM}$ L-glutamin, $0.01 \mathrm{M}$ HEPES and $10 \mathrm{ml} \mathrm{l}^{-1}$ antibiotic-antimycotic, and then extensively washed with pure L-15. The cells were then trypsinated and re-seeded (Day 0) and subjected to osteogenic differentiation medium composed of growth medium supplemented with $4 \mathrm{mM}$ $\mathrm{CaCl}_{2}, 10 \mathrm{mM} \beta$-glycerophosphate, $150 \mu \mathrm{M}$ L-ascorbic acid, $1 \mu \mathrm{M}$ 1,25-dihydroxyvitamin $\mathrm{D}_{3}$ and $10 \mathrm{nM}$ dexamethasone. Cells were harvested after 1,2 and $3 \mathrm{wk}$, as described in Ytteborg et al. (2010c). Cells were incubated at $12^{\circ} \mathrm{C}$ without $\mathrm{CO}_{2}$ and the medium was changed every second day. Their osteoblastic phenotype during differentiation has previously been described (Ytteborg et al. 2010c). Total RNA was isolated using an RNeasy Mini Kit and QIAshredder columns with the on-column RNase-Free DNase set (Qiagen), all in accordance with the manufacturer's protocols. The total RNA concentration and quality were determined by spectrophotometry (NanoDrop ND-1000 Spectrophotometer, NanoDrop Technologies). One microgram of total RNA was reversed transcribed to cDNA as described above. The experiment was repeated 4 times.

\section{Statistical analysis}

Statistical analysis was performed using a 2-tailed, unpaired Student's $t$-test. p-values $<0.05$ were considered statistically significant.

\section{RESULTS}

\section{Localization of decorin, biglycan and lumican in ND and FS vertebrae}

The distribution of the SLRPs was visualized by immunohistochemistry (Figs. 2 to 4). In non-deformed vertebrae, staining of all SLRPs (Fig. 2A decorin, Fig. 3A biglycan, Fig. 4A lumican) was detected in osteoblasts surrounding the vertebral endplates. Weaker staining was detected for all the SLRPs in regions where osteoblasts differentiate into mature osteoblasts, as seen in the intervertebral areas of the amphicoel growth zones. Staining was also detected in mesenchymale tissue above the osteoblast area. In addition, the chordocytes stained positive for all SLRPs, representing differentiated and mature cells of the notochord. However, no expression of SLRPs was obtained in proliferating cells of the notochord, the chordoblasts. Immunostaining of the SLRPs was also detected in hypertrophic chondrocytes at the ossification fronts in the arch centra (Fig. 2B decorin, Fig. 3B biglycan, Fig. 4B lumican). Some traces of staining could be detected in proliferating and resting chondrocytes, but this was not consistent between the different samples.

In vertebral fusions, the expression of SLRPs was extended to regions with aberrant cellular differentiation patterns (Ytteborg et al. 2010a). In the amphicoel growth zones, staining intensity increased (Fig. 2C decorin, Fig. 3C biglycan, Fig. 4C lumican). The expression of all 3 SLRPs was found at the border between arch centra and osteoblast growth zones (Fig. 2D decorin, Fig. 3D biglycan, Fig. 4D lumican).

\section{Protein expression of SLRPs in ND and FS vertebrae}

The expression of SLRPs was further verified by western blot. Protein cores with a molecular size of between 40 and $50 \mathrm{kDa}$ were found in ND and FS vertebrae (Fig. 5). Only one band of biglycan was detected in ND samples. An additional band of biglycan with a higher molecular weight was obtained 

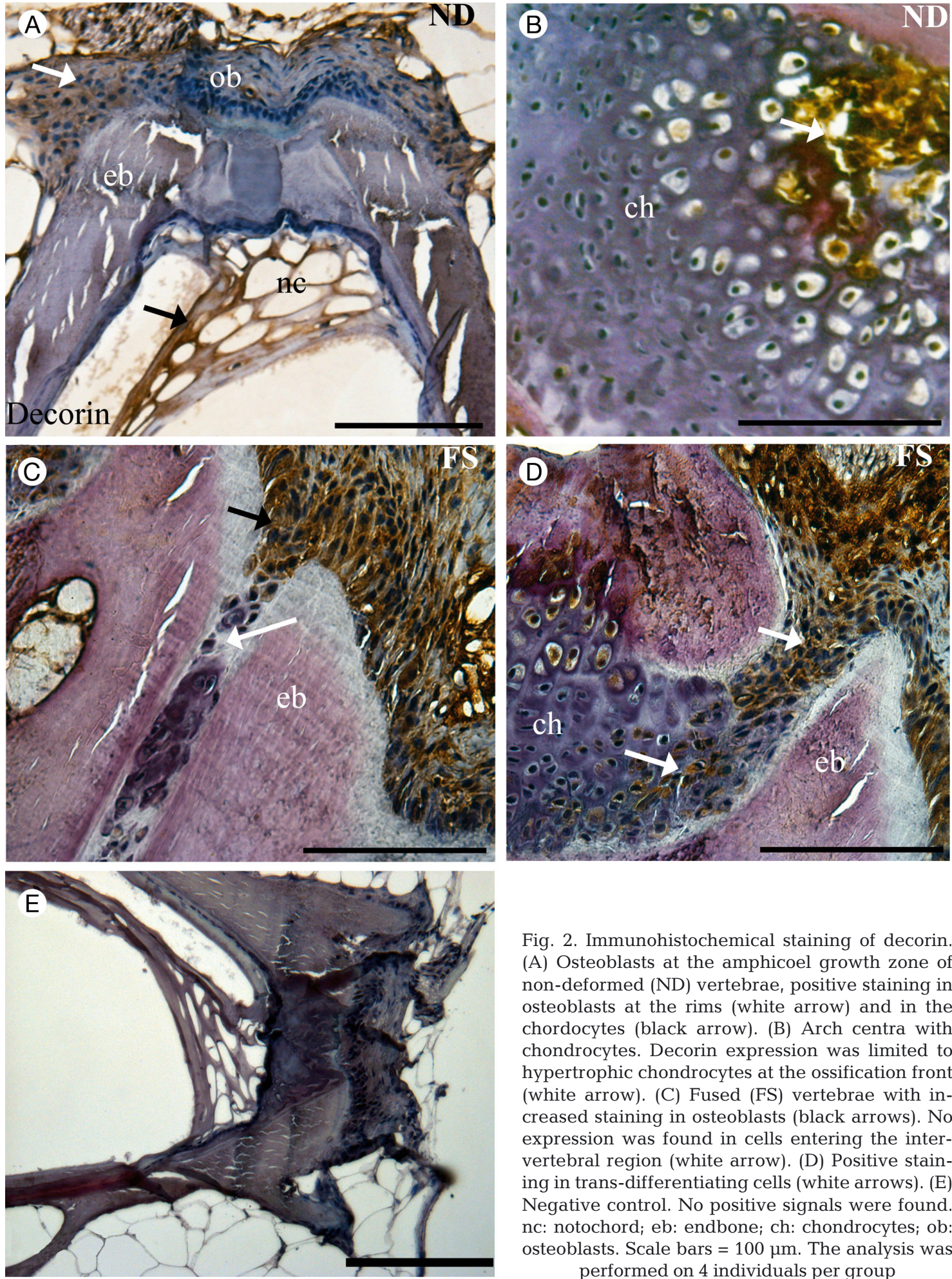

Fig. 2. Immunohistochemical staining of decorin. (A) Osteoblasts at the amphicoel growth zone of non-deformed (ND) vertebrae, positive staining in osteoblasts at the rims (white arrow) and in the chordocytes (black arrow). (B) Arch centra with chondrocytes. Decorin expression was limited to hypertrophic chondrocytes at the ossification front (white arrow). (C) Fused (FS) vertebrae with increased staining in osteoblasts (black arrows). No expression was found in cells entering the intervertebral region (white arrow). (D) Positive staining in trans-differentiating cells (white arrows). (E) Negative control. No positive signals were found. nc: notochord; eb: endbone; ch: chondrocytes; ob: osteoblasts. Scale bars $=100 \mu \mathrm{m}$. The analysis was performed on 4 individuals per group

from samples of FS vertebrae. Higher molecular SLRPs weights have has been detected during the mineralization phase of cells in vitro (Waddington et al. 2003).

\section{Transcription of SLRPs in vivo and in vitro}

The transcription of decorin, biglycan and lumican in ND and FS vertebrae was assessed by 

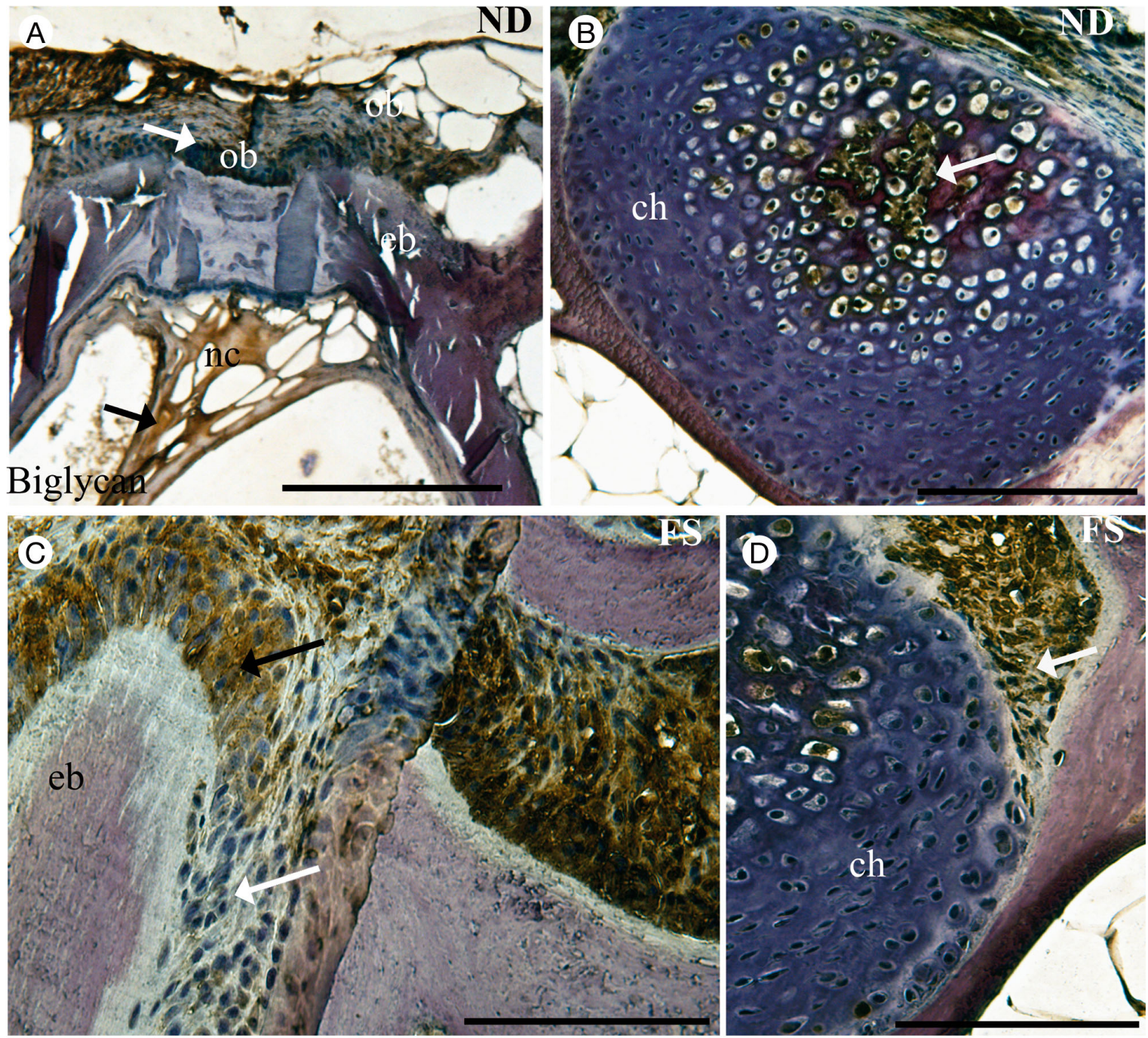

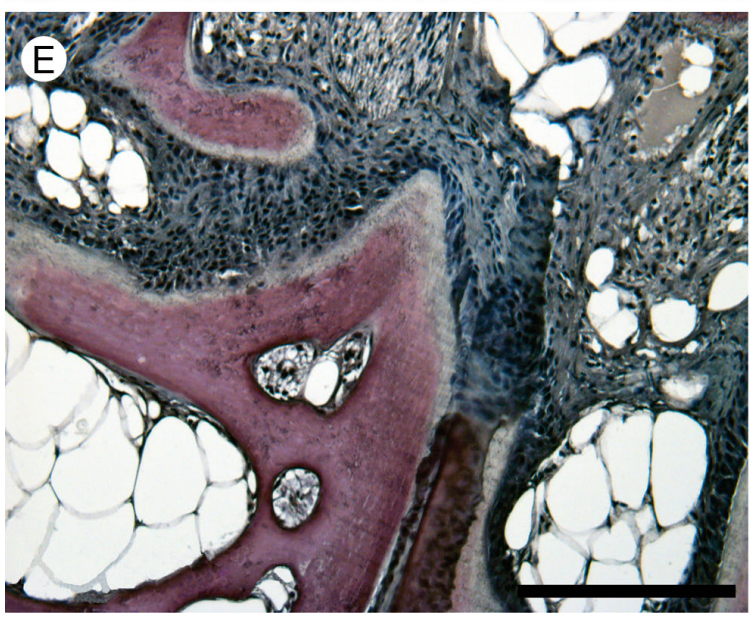

real-time qPCR. A different mRNA expression pattern of the SLRPs was observed in ND and FS vertebrae (Fig. 6A). Decorin was significantly up-regulated, whereas biglycan and lumican was
Fig. 3. Immunohistochemical staining of biglycan. (A) Osteoblasts at the amphicoel growth zone of non-deformed (ND) vertebrae showing positive staining (white arrow) and in chordocytes (black arrow). (B) Arch centra with chondrocytes. Biglycan expression was limited to hypertrophic chondrocytes at the ossification front (white arrow). (C) Vertebral fusion (FS) showing strong staining in the osteoblasts/trans-differentiating cells at the amphicoel growth zone (black arrow), but little staining in the cells in the intervertebral regions (white arrow). (D) Arch centra with chondrocytes, notice positive staining in the transdifferentiating cells between the arch centra and the amphicoel growth zone (white arrow). (E) Negative control. No positive signals were found. nc: notochord; eb: endbone; ch: chondrocytes; ob: osteoblasts. Scale bars $=100 \mu \mathrm{m}$. The analysis was performed on 4 individuals per group

significantly down-regulated in FS vertebrae. The mRNA expression of biglycan, decorin and lumican was also analyzed in an osteoblastic cell culture derived from mesenchymal cells from 

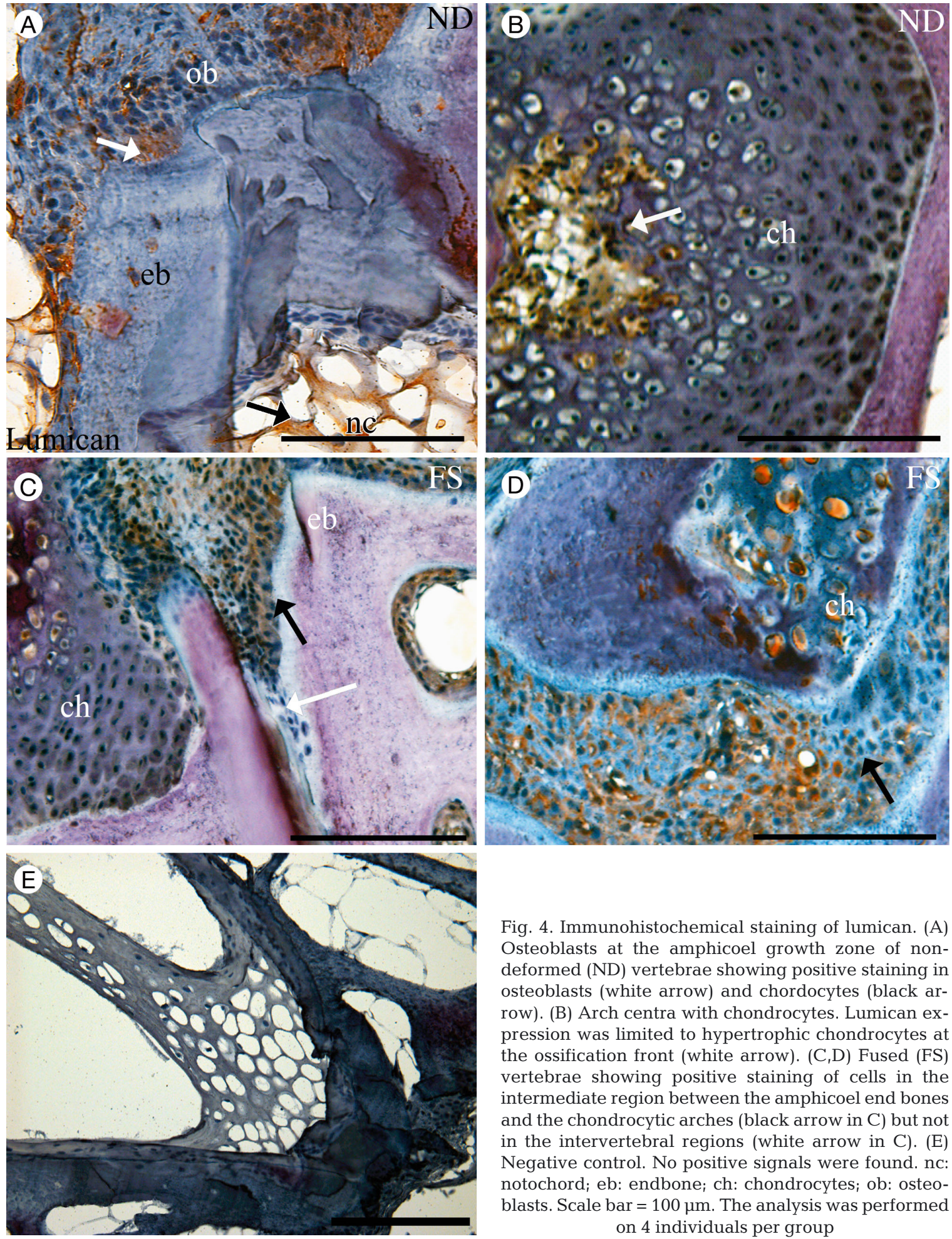

Fig. 4. Immunohistochemical staining of lumican. (A) Osteoblasts at the amphicoel growth zone of nondeformed (ND) vertebrae showing positive staining in osteoblasts (white arrow) and chordocytes (black arrow). (B) Arch centra with chondrocytes. Lumican expression was limited to hypertrophic chondrocytes at the ossification front (white arrow). (C,D) Fused (FS) vertebrae showing positive staining of cells in the intermediate region between the amphicoel end bones and the chondrocytic arches (black arrow in C) but not in the intervertebral regions (white arrow in $C$ ). (E) Negative control. No positive signals were found. nc: notochord; eb: endbone; ch: chondrocytes; ob: osteoblasts. Scale bar $=100 \mu \mathrm{m}$. The analysis was performed on 4 individuals per group

Atlantic salmon (Ytteborg et al. 2010c). A transcription pattern with increasing levels of decorin, biglycan and lumican was observed as the cells differentiated into osteoblast-like cells (Fig. 6B).
Decorin and biglycan showed a higher transcription during the early stages, Weeks 1 and 2, whereas lumican showed a pronounced increase in Week 3. 


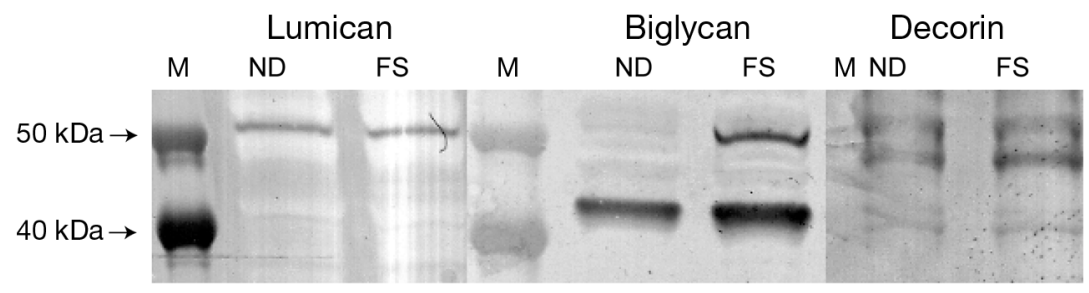

Fig. 5. Protein expression of small leucine-rich proteoclycans (SLRPs). Protein samples isolated from individuals of non-deformed $(\mathrm{ND} ; \mathrm{n}=5)$ and fused $(F S ; n=5)$ vertebrae were subjected to western blotting. M: protein markers. Molecular weights are indicated on the left side with arrows. Only one band of biglycan was detected in ND samples. Note the higher molecular weight of the biglycan band in the FS vertebra
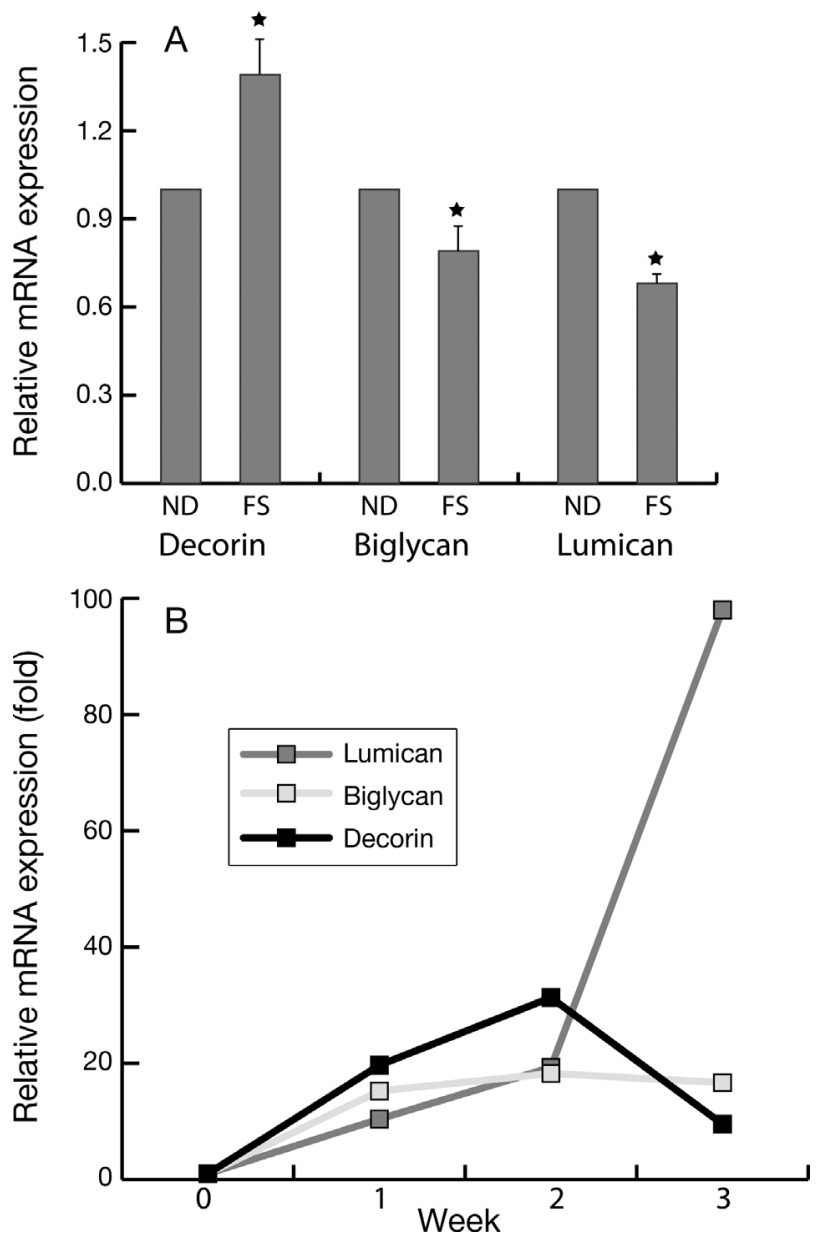

Fig. 6. Transcription of small leucine-rich proteoclycans (SLRPs). (A) In vivo transcription in non-deformed (ND) and fused (FS) vertebrae from 12 individuals. Decorin was upregulated, while biglycan and lumican down-regulated in FS samples compared with ND. Asterisks indicate significant difference $(\mathrm{p}<0.05)$ and black bars standard deviation. (B) In vitro transcription in osteoblasts during the differentiation stages Weeks 1-3. All transcripts increased during the first 3 wk compared with non-differentiated cells (Week 0). Lumican was highest expressed at Week 3, whereas biglycan and decorin peaked at Week 2. Levels of transcripts are relative to elongation factor 1 alpha $(\mathrm{EF}-1 \alpha)$, and data are presented as fold difference

\section{Structural properties of the endplates from ND and FS vertebrae}

FTIR analysis was used to measure mineral/matrix, collagen crosslinks, carbonation and crystallinity in the vertebral endplate of ND and FS vertebrae.

A significant difference in the maturation of collagen crosslinking was found, with less mature collagen crosslinking in the endplate of FS vertebrae compared with the endplate of ND vertebrae (Table 2). In addition, greater variability in spectra recorded in nearby areas of endplate of FS vertebrae was obtained (Fig. 7). This indicated more structural diversity in the endplate of FS vertebrae compared with ND vertebrae.

\section{DISCUSSION}

This study describes the expression and localization of biglycan, decorin and lumican in the vertebrae of a teleost, Atlantic salmon. The expression patterns at mRNA and protein levels were further compared in developing spinal fusions in order to study a possible role of SLRPs during pathogenesis.

Our data demonstrated a well-defined expression pattern of SLRPs in ND vertebrae and with temporal transcription in osteoblasts in vitro. The patterns indicate that SLRPs play a role in bone development in Atlantic salmon. The 3 SLRPs, decorin, biglycan and lumican, are all expressed in osteoblasts in the amphicoel growth zones in mature osteoblasts. In addition, all SLRPs were expressed in hypertrophic chondrocytes in the ossification fronts of the arches in normal developing vertebrae of Atlantic salmon. The finding that the SLRPs are expressed in regions where intramembraneous and endochondral ossification takes place hence suggests that they are involved in mineralization. In mammals, SLRPs have been detected in osteoblast and hypertrophic chondrocytes of limb long bones and in developing bone matrices (Bianco et al. 1990, Takagi et al. 2000, Alini \& Roughley 2001, Raouf et al. 2002, Yang et al. 2012) and appear to play a role related to establishing ossification fronts and matrix organization. All 3 proteins showed a molecular size between 40 and $50 \mathrm{kDa}$, which is in accordance with results from other teleosts (Tingbø et al. 2012) and mammals (Iozzo 1999, Pedersen et al. 2001) reflecting cross-species similarities. We found that SLRPs in Atlantic salmon 
Table 2. Fourier-transform infrared (FTIR) analyses of vertebral endplates $\left( \pm \mathrm{SD} ; \mathrm{n}=3\right.$ in each group). ${ }^{*}$ Significant difference between endbone of nondeformed (ND) and fused (FS) vertebrae $(p<0.05)$

\begin{tabular}{|lcccc|}
\hline Group & Mineral/matrix & Carbonated & Crosslinks & Crystallinity \\
\hline ND & $2.159 \pm 0.036$ & $0.076 \pm 0.001$ & $1.652 \pm 0.015$ & $1.014 \pm 0.001$ \\
FS & $2.233 \pm 0.054$ & $0.077 \pm 0.002$ & $1.563 \pm 0.031^{*}$ & $1.014 \pm 0.001$ \\
\hline
\end{tabular}

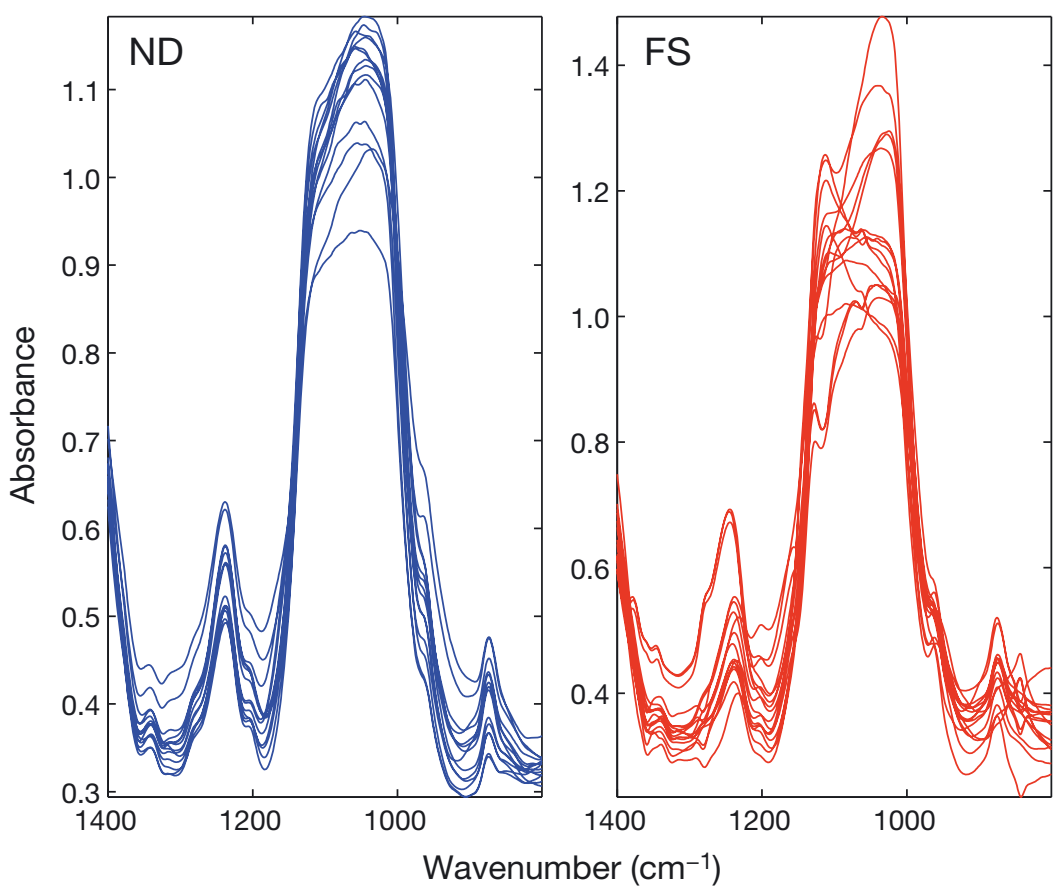

Fig. 7. Fourier-transform infrared (FTIR) spectra of non-deformed (ND) and fused (FS) vertebrae, measured from vertebrae endplates from 3 individuals in each group. Greater variability was obtained in spectra recorded in nearby areas of FS vertebrae endplates compared with ND vertebrae endplates. This indicated more structural diversity of the FS vertebrae endplate

dance with mammals, where lumican has been demonstrated in vitro to be abundantly expressed at mature stages and not during the proliferation stage, being one of the matrix proteins most highly expressed by mature osteoblasts (Raouf \& Seth 2002, Raouf et al. 2002). Thus, our in vitro findings are in line with the in vivo findings, where all 3 proteins were found to have higher expression in mature osteoblasts. Hence, all in all, we suggest that the SLRPs have specific roles in relation to the maturation of osteoblasts and chondrocytes in Atlantic salmon.

In FS vertebrae, the expression of the SLRPs was extended to areas undergoing pathological changes. The SLRPs were found in cells at the border between the arch centra and osteoblast growth zones during the fusion process, areas previously described harboring cells in a trans-differentiating state (Ytteborg et al. 2010a). In Ytteborg et al. (2010a) it was further found that these cells produced mineralized matrix, causing ectopic mineralization of the vertebrae. Thus, positive signals of biglycan, decorin and lumican in these regions support our suggestion of their involvement in mineralization. Likewise, in mammals, it has been suggested that ECM components such as SLRPs could play important roles in controlling cell fate and func-

osteoblasts are increasingly transcribed during differentiation in vitro, as described in mammalian osteoblast cell lines (Raouf \& Seth 2002, Raouf et al. 2002, Mochida et al. 2003, Waddington et al. 2003, Chen et al. 2005, Parisuthiman et al. 2005). The transcription of the SLRPs followed the transcription pattern of other important genes in osteoblast development, such as bone morphogenic protein, collagen type 1 and alkaline phosphatase, previously described for these cells (Ytteborg et al. 2010c). Biglycan control signaling pathways regulating the osteogenic program (Berendsen et al. 2011). The ability of bone marrow stromal cells to differentiate into bone-forming cells in vivo correlated with decorin expression (Larsen et al. 2010). In our study, transcription of lumican increased at later differentiation stages, compared with decorin and biglycan. This is in accor- tion, causing them to form ectopic bone (Ameye et al. 2002, Kilts et al. 2009). High expression of biglycan has been observed in ectopic chondro-ossification sites (Lui et al. 2012). Sustained and increased expression of both decorin and biglycan have been observed in tendons of an animal model mimicking calcified tendinopathy (Lui et al. 2012).

We further demonstrated that biglycan was expressed with an additional variant with a higher molecular weight in vertebral fusions, indicating modifications of biglycan at the protein level. SLRPs with a higher molecular weight have been detected during the mineralization of cells in vitro (Waddington et al. 2003), and it has been suggested that this higher weight form is a regulatory mechanism important for the mineralization process. The modification of biglycan in vertebral fusions, in contrast to decorin 
and lumican, could indicate different regulatory mechanisms of the SLRPs in bone development of Atlantic salmon. Our antibody recognized both forms, and we therefore do not know whether the distribution of both variants is the same. Further studies have to be performed to reveal the expression of the higher molecular weight band of biglycan in the vertebrae fusions. In our study, the SLRPs differed at the mRNA level between ND and FS vertebrae. Decorin transcription was increased, whereas lumican and biglycan transcription decreased. Thus, both translation and transcription suggested an involvement of the SLRPs in the development of vertebral fusions. Studies in vitro with overexpression of decorin or underexpression of biglycan resulted in a reduced mineralization (Mochida et al. 2003, Parisuthiman et al. 2005). FTIR revealed greater variability in spectra recorded in nearby areas of the amphicoel endplates of FS vertebrae, indicating more structural diversity of FS vertebrae compared with ND vertebrae. Interestingly, FTIR data showed a significant difference in maturation of collagen crosslinks that are important in collagen organization. The SLRPs play specific roles in fibrillogenesis, forming different collagen networks and fibril bundle phenotypes (Svensson et al. 1995, Danielson et al. 1997, Matheson et al. 2005, Kalamajski \& Oldberg 2010). Studies have also demonstrated that decorin play a direct role in regulating collagen crosslinking in vitro (e.g. Seidler et al. 2005). Collagen organization and collagen crosslinks further regulate mineralization, due to the availability of minerals to the template (Gerstenfeld et al. 1993, Yamauchi \& Katz 1993, Pornprasertsuk et al. 2004, 2005). The mechanism involved in regulation of collagen crosslinking is unknown; however, it has been speculated that SLRPs may act as co-receptors for lysyl oxidase or sterically hinder the binding of lysyl oxidase to the collagen target (Kalamajski \& Oldberg 2010). The difference found by FTIR in spinal fusions supports our overall findings of a disturbed matrix organization and altered mineralization pattern during the pathological development.

Our study has shown that SLRPs are important components to study with respect to both normal bone development in teleosts and matrix organization and mineralization during the pathological development of vertebral fusions.

Acknowledgements. This study was supported by the Norwegian Research Council, project number 172483/S40 and the EU (COLL-CT-2005-012451, FINE FISH). We thank Nancy Pleshko for help and advice with the FTIR analysis.

\section{LITERATURE CITED}

Alini M, Roughley PJ (2001) Changes in leucine-rich repeat proteoglycans during maturation of the bovine growth plate. Matrix Biol 19:805-813

Ameye L, Aria D, Jepsen K, Oldberg A, Xu TS, Young MF (2002) Abnormal collagen fibrils in tendons of biglycan/ fibromodulin-deficient mice lead to gait impairment, ectopic ossification, and osteoarthritis. FASEB J 16: 673-680

Baeverfjord G (2003) Short tail deformities in Atlantic salmon-effect of freshwater production temperature. Beyond monoculture. EAS Spec Publ 33:121-122

Berendsen AD, Fisher LW, Kilts TM, Owens RT, Robey PG, Gutkind JS, Young MF (2011) Modulation of canonical Wnt signaling by the extracellular matrix component biglycan. Proc Natl Acad Sci USA 108:17022-17027

> Bianco P, Fisher L, Young M, Termine J, Robey P (1990) Expression and localization of the two small proteoglycans biglycan and decorin in developing human skeletal and non-skeletal tissues. J Histochem Cytochem 38:1549-1563

Boskey A, Camacho NP (2007) FT-IR imaging of native and tissue-engineered bone and cartilage. Biomaterials 28: 2465-2478

Boskey AL, Spevak L, Doty SB, Rosenberg L (1997) Effects of bone CS-proteoglycans, DS-decorin, and DS-biglycan on hydroxyapatite formation in a gelatin gel. Calcif Tissue Int 61:298-305

Chen XD, Bian XP, Teslovich TM, Stephan DA, Young MF (2005) Dissection of the sets of genes that control the behavior of biglycan-deficient pre-osteoblasts using oligonucleotide microarrays. Bone 37:192-203

Corsi A, Xu T, Chen X, Boyde A and others (2002) Phenotypic effects of biglycan deficiency are linked to collagen fibril abnormalities, are synergized by decorin deficiency, and mimic Ehlers-Danlos-like changes in bone and other connective tissues. J Bone Miner Res 17: 1180-1189

> Danielson KG, Baribault H, Holmes DF, Graham H, Kadler KE, Iozzo RV (1997) Targeted disruption of decorin leads to abnormal collagen fibril morphology and skin fragility. J Cell Biol 136:729-743

Gentili C, Cancedda R (2009) Cartilage and bone extracellular matrix. Curr Pharm Des 15:1334-1348

Gerstenfeld LC, Riva A, Hodgens K, Eyre DR, Landis WJ (1993) Post-translational conrtol of collagen fibrillogenesis in mineralizing cultures of chick osteoblasts. J Bone Miner Res 8:1031-1043

> Iozzo RV (1998) Matrix proteoglycans: from molecular design to cellular function. Annu Rev Biochem 67: 609-652

> Iozzo RV (1999) The biology of the small leucine rich proteoglycans. J Biol Chem 274:18843-18846

> Kalamajski S, Oldberg $\AA$ (2010) The role of small leucinerich proteoglycans in collagen fibrillogenesis. Matrix Biol 29:248-253

Kilts T, Ameye L, Picard F, Ono M and others (2009) Potential roles for the small leucine-rich proteoglycan biglycan and fibromodulin in ectopic ossification of tendon induced by exercise and in modulating rotard performance. Scand J Med Sci Sports 19:536-546

Koumans J, Akster H, Osse J (1990) In vitro culturing of carp (Cyprinus carpio L) myosatellite cells as a tool to study growth of fish muscle. J Muscle Res Cell Motil 11:87 
Larsen KH, Frederiksen CM, Burns JS, Abdallah BM, Kassem M (2010) Identefying a molecular phenotype for bone marrow stromal cells with in vivo bone-forming capacity. J Bone Miner Res 25:796-808

Lui PPY, Cheuck YC, Lee YW, Chan KM (2012) Ectopic chondro-ossification and erroneous extracellular matrix deposition in a tendon window injury model. J Orthop Res 30:37-46

Matheson S, Larjava H, Hakkinen L (2005) Distinctive localization and function for lumican, fibromodulin and decorin to regulate collagen fibril organization in periodontal tissues. J Periodontal Res 40:312-324

> Mochida Y, Duarte WR, Tanzawa H, Paschalis EP, Yamauchi M (2003) Decorin modulates matrix mineralization in vitro. Biochem Biophys Res Commun 305:6-9

> Nikitovic D, Aggelidakis J, Young MF, Iozzo RV, Karamanos NK, Tzanakakis GN (2012) The biology of small leucinerich proteoglycans in bone pathophysiology. J Biol Chem 287:33926-33933

> Nuka S, Zhou W, Henry SP, Gendron CM and others (2010) Phenotypic characterization of epiphycan-deficient and epiphycan/biglycan double-deficient mice. Osteoarthritis Cartilage 18:88-96

Parisuthiman D, Mochida Y, Duarte WR, Yamauchi M (2005) Biglycan modulates osteoblast differentiation and matrix mineralization. J Bone Miner Res 20:1878-1886

> Pedersen M, Kulseth M, Kolset S, Velleman S, Eggen K (2001) Decorin and fibromodulin expression in two bovine muscles ( $M$. semitendinosus and $M$. psoas major) differing in texture. J Muscle Foods 12:1-17

> Pedersen ME, Takle H, Ytteborg E, Veiseth-Kent E and others (2011) Matrilin-1 expression is increased in the vertebral column of Atlantic salmon (Salmo salar L.) individuals displaying spinal fusions. Fish Physiol Biochem 37:821-831

Pihlajaniemi T, Heikkinen A, Keski-Filppula R, Tu H, Fox MA, Sanes JR (2009) Extracellular matrix in bone and cartilage pathologies: regulatory and structural roles of collagen XIII in bone and muscle. FEBS J 276:32

Pornprasertsuk S, Duarte WR, Mochida Y, Yamauchi M (2004) Lysyl hydroxylase-2b directs collagen cross-linking pathways in MC3T3-E1 cells. J Bone Miner Res 19: 1349-1355

Pornprasertsuk S, Duarte WR, Mochida Y, Yamauchi M (2005) Overexpression of lysyl hydroxylase-2b leads to defective collagen fibrillogenesis and matrix mineralization. J Bone Miner Res 20:81-87

Raouf A, Seth A (2002) Discovery of osteoblast-associated genes using cDNA microarray. Bone 30:463-471

Raouf A, Ganss B, McMahon C, Vay C, Roughley P, Seth A (2002) Lumican is a major proteoglycan component of the bone matrix. Matrix Biol 21:361-367

Schaefer L, Iozzo RV (2008) Biological functions of the small leucine-rich proteoglycans: from genetics to signal transduction. J Biol Chem 283:21305-21309

> Seidler DG, Schaefer L, Robenek H, Iozzo RV, Kresse H, Schonherr E (2005) A physiologic three-dimensional cell culture system to investigate the role of decorin in matrix organisation and cell survival. Biochem Biophys Res Commun 332:1162-1170

> Svensson L, Heinegard D, Oldberg Å (1995) Decorin-bind-

Editorial responsibility: Catherine Collins,

Aberdeen, UK ing sites for collagen type I are mainly located in leucinerich repeats 4-5. J Biol Chem 274:20712-20716

Takagi M, Kamiya N, Urushizaki T, Tada Y, Tanaka H (2000) Gene expression and immunohistochemical localization of biglycan in association with mineralization in the matrix of epiphyseal cartilage. Histochem J 32: 175-186

- Tingbø MG, Pedersen ME, Kolset SO, Enersen G, Hannesson KO (2012) Lumican is a major small leucine-rich proteoglycan (SLRP) in Atlantic cod (Gadus morhua L.) skeletal muscle. Glycoconj J 29:13-23

> Torgersen JS, Takle H, Andersen Ø (2009) Localization of mRNAs and proteins in methyl methacrylate embedded tissues. J Histochem Cytochem 57:825-830

> Vegusdal A, Ostbye TK, Tran TN, Gjoen T, Ruyter B (2004) beta-oxidation, esterification, and secretion of radiolabeled fatty acids in cultivated Atlantic salmon skeletal muscle cells. Lipids 39:649-658

> Waddington RJ, Roberts HC, Sugars RV, Schönherr E (2003) Differential roles for small leucine-rich proteoglycans in bone formation. Eur Cell Mater 6:12-21

> Witten PE, Gil-Martens L, Hall BK, Huysseune A, Obach A (2005) Compressed vertebrae in Atlantic salmon Salmo salar: evidence for metaplastic chondrogenesis as a skeletogenic response late in ontogeny. Dis Aquat Org 64:237-246

> Witten PE, Obach A, Huysseune A, Baeverfjord G (2006) Vertebrae fusion in Atlantic salmon (Salmo salar): development, aggravation and pathways of containment. Aquaculture 258:164-172

Witten PE, Gil-Martens L, Huysseune A, Takle H, Hjelde K (2009) Towards a classification and an understanding of developmental relationships of vertebral body malformations in Atlantic salmon (Salmo salar L.). Aquaculture 295:6-14

Yamauchi M, Katz E (1993) The post-translational chemistry and molecular packing of mineralizing tendon collagens. Connect Tissue Res 29:81-98

Yang $\mathrm{CH}_{1}$ Culshaw GJ, Liu MM, Lu CC, French AT, Clements DN, Corcoran BM (2012) Canine tissuespecific expression of multiple small leucine rich proteoglycans. Vet J 193:374-380

> Ytteborg E, Baeverfjord G, Hjelde K, Torgersen J, Takle H (2009) Temperature affects the expression of genes involved in the mineralization process of the spinal column in Atlantic salmon (Salmo salar). Bone 44: S341-S342

Ytteborg E, Torgersen J, Baeverfjord G, Takle H (2010a) Morphological and molecular characterization of developing vertebral fusions using a teleost model. BMC Physiol 10:13

> Ytteborg E, Torgersen JS, Pedersen ME, Baeverfjord G, Hannesson KO, Takle H (2010b) Remodeling of the notochord during development of vertebral fusions in Atlantic salmon (Salmo salar). Cell Tissue Res 342:363-376

Ytteborg E, Vegusdal A, Witten PE, Berge GM, Takle H, Ostbye TK, Ruyter B (2010c) Atlantic salmon (Salmo salar) muscle precursor cells differentiate into osteoblasts in vitro: polyunsaturated fatty acids and hyperthermia influence gene expression and differentiation. Biochim Biophys Acta 1801:127-137

Submitted: January 7, 2013; Accepted: June 23, 2013 Proofs received from author(s): September 10, 2013 